\title{
Surgical case of intracranial osteoma arising from the falx
}

\author{
SATORU TAKEUCHI ${ }^{1,2}$, ROKUYA TANIKAWA ${ }^{2}$, TOSHIYUKI TSUBOI ${ }^{2}$, KOSUMO NODA $^{2}$, \\ SHIRO MIYATA $^{2}$, NAKAO OTA ${ }^{2}$, FUMIHIRO HAMADA ${ }^{2}$ and HIROYASU KAMIYAMA ${ }^{2}$ \\ ${ }^{1}$ Department of Neurosurgery, National Defense Medical College, Saitama 359-8513; \\ ${ }^{2}$ Department of Neurosurgery, Teishinkai Hospital, Hokkaido 065-0033, Japan
}

Received April 6, 2015; Accepted June 2, 2016

DOI: $10.3892 / \mathrm{ol} .2016 .4833$

\begin{abstract}
Intracranial osteomas completely unrelated to osseous tissues are extremely rare. In the present study, the case of a 40-year-old female who presented with persistent headache is reported. Computed tomography (CT) and bone window $\mathrm{CT}$ revealed an ossified lesion in the frontal area. Fast imaging employing steady-state acquisition (FIESTA)/CT venography fusion imaging demonstrated that the mass was located just below the superior sagittal sinus and cortical veins, and had adhered partially to these veins. Surgery achieved complete tumor removal with preservation of the cortical veins and superior sagittal sinus. The histological examination findings were compatible with osteoma. The present postoperative course was uneventful. The present rare case of intracranial osteoma originating from the falx was successfully treated surgically. Preoperative FIESTA/CT venography fusion imaging was very useful to demonstrate adhesion between the tumor mass and the superior sagittal sinus and cortical veins.
\end{abstract}

\section{Introduction}

Osteomas are benign neoplasms consisting of mature normal osseous tissue (1). Osteomas of the head often arise from the periosteum of the skull, sinuses and mandible (1). Intracranial osteomas are rare, and usually arise from the inner table of the skull (1). Intracranial osteomas completely unrelated to osseous tissues are extremely rare, and are classified into intraparenchymal osteomas and dural osteomas (1). Despite a series of 10 autopsy cases of dural osteomas, the clinical features and surgical strategy remain unclear (2). The present report describes a rare surgical case of intracranial osteoma

Correspondence to: $\mathrm{Dr}$ Satoru Takeuchi, Department of Neurosurgery, National Defense Medical College, 3-2 Namiki, Tokorozawa, Saitama 359-8513, Japan

E-mail: s.takeuchi@room.ocn.ne.jp

Abbreviations: CT, computed tomography; FIESTA, fast imaging employing steady-state acquisition

Key words: osteoma, intracranial, falx, surgery arising from the falx, and discusses the clinical features, intraoperative findings and management strategy of this disease.

\section{Case report}

In March 2014, a 40-year-old female was admitted to Teishinkai Hospital (Hokkaido, Japan) due to persistent headache. On admission, neurological examination revealed no abnormalities. Skull radiography revealed a radiopaque lesion in the frontal area (Fig. 1A and B), while computed tomography (CT) and bone window $\mathrm{CT}$ revealed an ossified lesion in the frontal area (Fig. 1C and D). Magnetic resonance imaging revealed a mass appearing as hyperintense on both T1- and T2-weighted images (Fig. 2). Three-dimensional CT venography (Fig. 3A and B) and fast imaging employing steady-state acquisition (FIESTA)/CT venography fusion imaging (Fig. 3C) demonstrated that the mass was located just below the superior sagittal sinus and cortical veins, and had adhered partially to these veins. The provisional diagnosis was calcified falx meningioma or osteoma originating from the falx. Bilateral frontal craniotomy was performed. Opening of the dura revealed that the mass was attached to the falx in the mesial frontal lobes extra-axially, without dural attachment. The hard bony mass was removed with a drill and a rongeur (Fig. 4A). The cortical veins and superior sagittal sinus were preserved completely. Postoperative CT demonstrated complete tumor resection without complication (Fig. 4B). Histological examination (hematoxylin and eosin staining) revealed lamellated bony trabeculae lined with osteoblasts, and the intertrabecular marrow spaces occupied by adipose tissue. These findings are compatible with osteoma (Fig. 5). The postoperative course was uneventful, and the symptoms disappeared after surgery. The patient was discharged 12 days after surgery without neurological deficit. The patient was followed up for 12 months without any recurrence.

Written informed consent was obtained from the patient for publication of the present case report and any accompanying images.

\section{Discussion}

Intracranial osteomas are rare, with only 12 surgical cases of osteomas without association with the bone being reported in the literature so far, including the present case, as summarized in Table I (3-13). The male to female ratio was 1:2, and the 

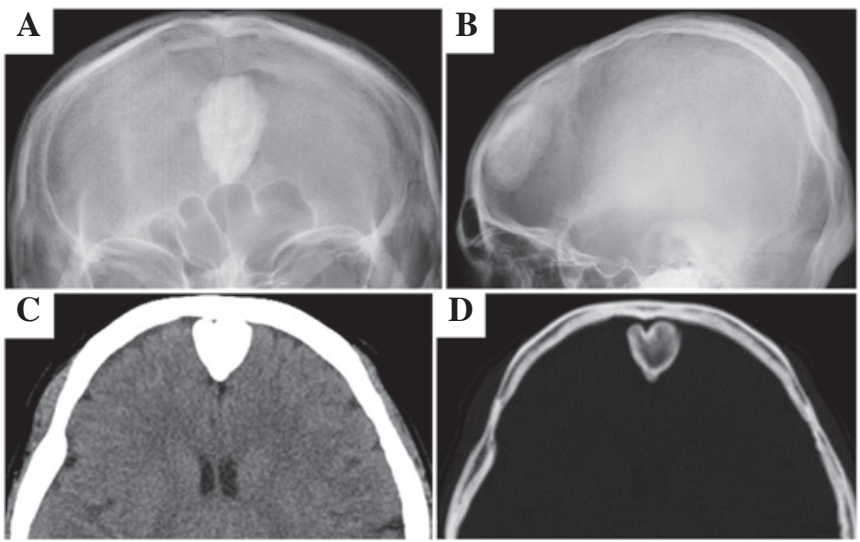

Figure 1. Skull radiographs of (A) anteroposterior and (B) lateral view revealed a radiopaque lesion in the frontal area. (C) CT scan and (D) bone window CT imaging revealed an ossified lesion in the frontal area. CT, computed tomography.
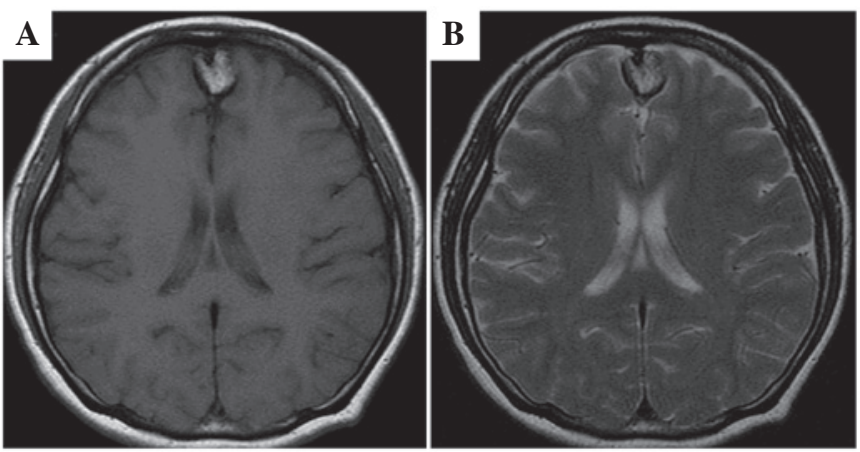

Figure 2. (A) T1- and (B) T2-weighted magnetic resonance images revealed a mass appearing as hyperintense.

age of the patients was $16-64$ years (mean, 39.8 years). The most common symptom was headache, which occurred in 8 of the 12 cases. The tumors were described as 'completely surrounded by cerebral tissue', with 'no connections with the dura or the skull', or 'covered with the arachnoid membrane' in 5 cases. The tumors were attached to the inner dural surface in 5 cases, and to the falx in 2 cases, indicating dural osteomas. Therefore, the present case is the second surgical case of intracranial osteoma originated from the falx. The outcome was good in 10 patients, whereas 1 patient succumbed to disease. Venous infarction developed in 1 case (10).

Intracranial osteoma usually has a wide base and grows inward as an expanding mass with a well-defined border (4). The present case occurred as a nodule with a narrow neck attached to the falx, similar to a previous case (13). The pathogenesis of osteoma without bone involvement is still unknown. In the present case, new bone generation from the falx is a possible cause, since the meninges may function as the periosteum of the inner table of the skull $(2,5,8)$. A history of head trauma has been noted in patients with intracranial osteomas. Therefore, head trauma may be one of the trigger mechanisms of intracranial osteoma (14); however, the present patient had no such history.

No standardized treatment algorithm has been established for this condition. If the patient is asymptomatic, conservative therapy is one of the choices, since the natural history of
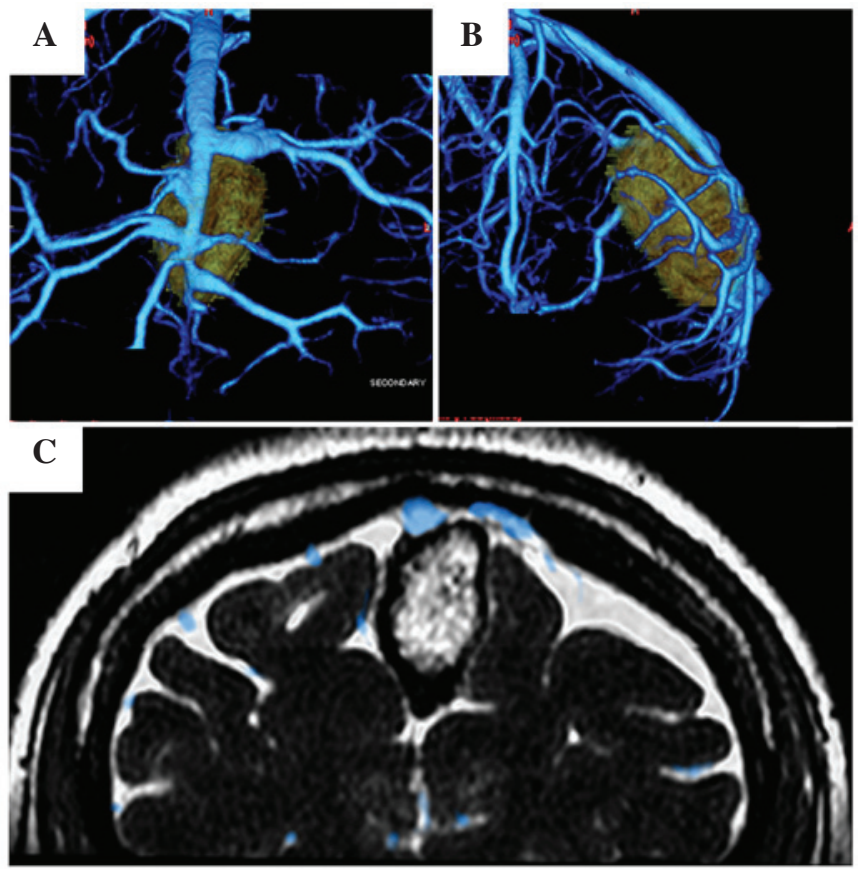

Figure 3. Three-dimensional CT venograms of (A) anteroposterior and (B) lateral view, and (C) fast imaging employing steady-state acquisition/CT venography fusion imaging demonstrated that the mass was located just below the superior sagittal sinus and cortical veins, and had adhered partially to these veins. CT, computed tomography.
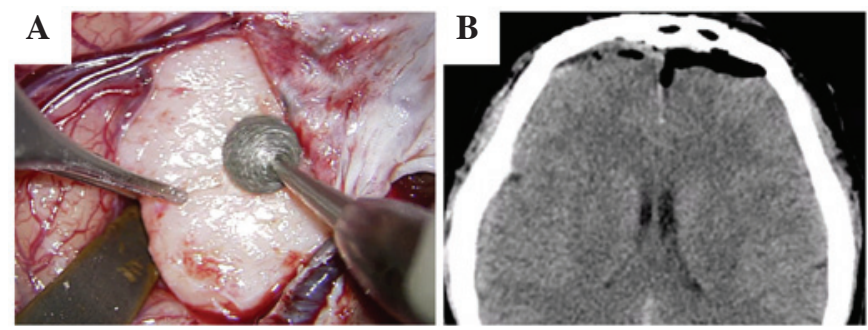

Figure 4. (A) Intraoperative image of the tumor attached to the falx, which was removed with a $4 \mathrm{~mm}$ diamond drill and a rongeur. The cortical veins and superior sagittal sinus were preserved completely. (B) Postoperative computed tomography scan demonstrated complete tumor resection without complications.

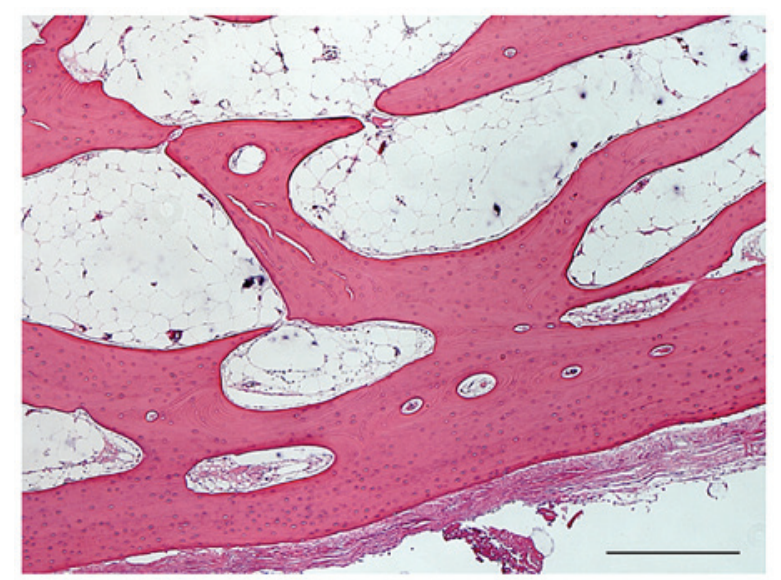

Figure 5. Photomicrograph revealing lamellated bony trabeculae lined with osteoblasts and intertrabecular marrow spaces occupied by adipose tissue, which is compatible with osteoma. Hematoxylin and eosin staining; bar size, $100 \mu \mathrm{m}$. 


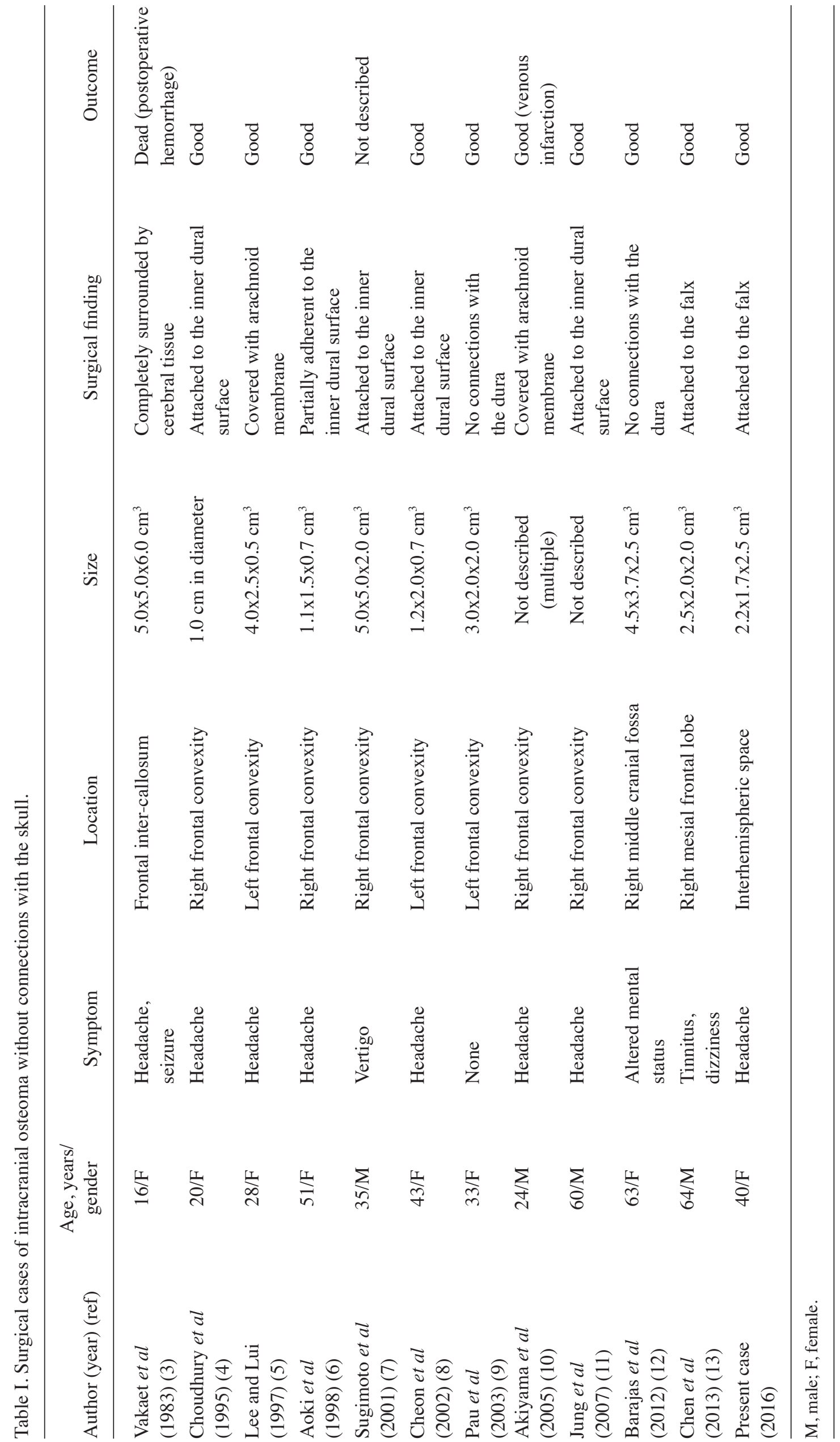


intracranial osteomas is more benign than that of osteomas of the frontal sinuses (3). The present patient complained of intractable headache, which could be resolved by surgical tumor removal, since such pain is considered to be due to irritation and compression of the adjacent dural membrane (10). Therefore, surgery was performed in the present case, and the pain disappeared completely.

Surgeons should pay particular attention to possible adhesion of intracranial osteoma to the blood vessels. In a previous case, the osteoma had adhered tightly to the superficial cortical veins, which were sacrificed, thus leading to postoperative venous congestion (10). In the present case, preoperative FIESTA/CT venography fusion imaging was very useful to demonstrate that the mass was located just below the superior sagittal sinus and cortical veins, and had adhered partially to these veins. Complete preservation of these structures was carefully achieved during surgery. Reconstruction via saphenous vein graft and patch is recommended if the superior sagittal sinus or cortical vein is injured $(15,16)$.

In conclusion, the present case of intracranial osteoma originating from the falx was successfully treated surgically. Preoperative FIESTA/CT venography fusion imaging was very useful to demonstrate adhesion between the mass and the superior sagittal sinus and cortical veins.

\section{References}

1. Haddad FS, Haddad GF and Zaatari G: Cranial osteomas: Their classification and management. Report on a giant osteoma and review of the literature. Surg Neurol 48: 143-147, 1997.

2. Fallon MD, Ellerbrake D and Teitelbaum SL: Meningeal osteomas and chronic renal failure. Hum Pathol 13: 449-453, 1982.
3. Vakaet A, De Reuck J, Thiery E and vander Eecken H: Intracerebral osteoma: A clinicopathologic and neuropsychologic case study. Childs Brain 10: 281-285, 1983.

4. Choudhury AR, Haleem A and Tjan GT: Solitary intradural intracranial osteoma. Br J Neurosurg 9: 557-559, 1995.

5. Lee ST and Lui TN: Intracerebral osteoma: Case report. Br J Neurosurg 11: 250-252, 1997.

6. Aoki H, Nakase H and Sakaki T: Subdural osteoma. Acta Neurochir (Wien) 140: 727-728, 1998.

7. Sugimoto K, Nakahara I, Nishikawa M, Tanaka M, Terashima T, Yanagihara $\mathrm{H}$ and Hayashi J: Osteoma originating in the dura: A case report. No Shinkei Geka 29: 993-996, 2001 (In Japanese).

8. Cheon JE, Kim JE and Yang HJ: CT and pathologic findings of a case of subdural osteoma. Korean J Radiol 3: 211-213, 2002.

9. Pau A, Chiaramonte G, Ghio G and Pisani R: Solitary intracranial subdural osteoma: Case report and review of the literature. Tumori 89: 96-98, 2003.

10. Akiyama M, Tanaka T, Hasegawa Y, Chiba S and Abe T: Multiple intracranial subarachnoid osteomas. Acta Neurochir (Wien) 147: 1085-1089, 2005.

11. Jung TY, Jung S, Jin SG, Jin YH, Kim IY and Kang SS: Solitary intracranial subdural osteoma: Intraoperative findings and primary anastomosis of an involved cortical vein. J Clin Neurosci 14: 468-470, 2007.

12. Barajas RF Jr, Perry A, Sughrue M, Aghi M and Cha S: Intracranial subdural osteoma: A rare benign tumor that can be differentiated from other calcified intracranial lesions utilizing MR imaging. J Neuroradiol 39: 263-266, 2012

13. Chen SM, Chuang CC, Toh CH, Jung SM and Lui TN: Solitary intracranial osteoma with attachment to the falx: A case report. World J Surg Oncol 11: 221, 2013.

14. Dukes HT and Odom GL: Discrete intradural osteoma. Report of a case. J Neurosurg 19: 251-253, 1962.

15. Hakuba A, Huh CW, Tsujikawa S and Nishimura S: Total removal of a parasagittal meningioma of the posterior third of the sagittal sinus and its repair by autogenous vein graft. Case report. J Neurosurg 51: 379-382, 1979.

16. Murata J, Sawamura Y, Saito $\mathrm{H}$ and Abe $\mathrm{H}$ : Resection of a recurrent parasagittal meningioma with cortical vein anastomosis: Technical note. Surg Neurol 48: 592-595; discussion 595-597, 1997. 